\title{
Research of hot air blower de-icing technology for
}

\section{wind turbine blade}

\author{
Yongshui LUO ${ }^{1, a}$, Jian LIU 1 , Qi CHEN ${ }^{1}$, Minqiang ZHOU ${ }^{1}$, Heng ZHANG ${ }^{1}$ \\ 'Zhejiang Windey Co., Ltd, State Key Laboratory of Wind Power System, Hangzhou 310012, China \\ aluoys@chinawindey.com
}

Keywords: wind power; wind turbine blade; hot air blower de-icing technology; temperature rise Abstract. Aiming at ice accretion on wind turbine blade causes power production loss and affects its normal performance safety, a novel hot air blower de-icing technique for blades was researched. The numerical simulation for the whole profile of blades was applied, and flow distribution of the blade and heat transfer between inner blade and outside were analyzed. Temperature and heat distribution on blade cavity and leading edge were measured in constant temperature laboratory, and compared the simulation and experiment data. The results indicated that the ventilation pipe design of hot air blower de-icing system contributed to the heat accumulation around blade leading edge, and the effect of heat transfer on the four key-point sections were significant. Particularly, the deviation of temperature rise on leading edge between the simulation value $(9 \mathrm{~K} \sim 19 \mathrm{~K})$ and the test temperature value $(11 \mathrm{~K} \sim 16.8 \mathrm{~K})$ was only $8.5 \%$, which illustrated that it reached the requirement of de-icing from the baffle to the blade tip. The deviation between temperature value and average value of three blades leading edge was within 9.5\%, which proved high consistency of data and the heat distribution of blade leading edge was reasonable. The effectiveness of hot air blower de-icing technology was verified, which was propelling the development of wind turbine application in cold weather conditions.

\section{Introduction}

The new model of wind industry that is "low wind speed wind turbine, proximity to the power grid, local consumption ${ }^{[1,2]}$, is prevailing in China, the southern region (Yunnan, Guizhou, Sichuan, Hubei and Hunan etc.) have built more and more wind farms. Climate characteristic of the southern region is the wind resources is best in winter, but the temperature is low (generally around 10 degrees below zero), humidity (higher than $85 \% \mathrm{RH}$ ), the wind turbine is very easy to freeze.

The blades of wind turbine are key components of the power generation efficiency, the icing of the blades will not only increase the additional load, reduce the service life of components, and seriously affect the stability and safety of the unit; and the ice structure will change the aerodynamic characteristics of the blade, reduce the generating efficiency of the unit, and when the ice files away from the blade due to the centrifugal force, cause obvious security risks on the unit itself and field personnel $^{[3-5]}$.

The research of the wind turbine blades' anti-icing / de-icing technology is mainly in the following directions at home and abroad ${ }^{[6,7,8]}$ : A. Coating technology program, improving surface temperature by using black paddle, but only the sunny day can gather temperature to melt ice, and high temperature will affect the surface material of the blade, has a low adaptability; spraying the hydrophobic chemical reagent to prevent the rain from freezing has the weaknesses of short action time, large amount and environmental pollution. B. Mechanical de-icing program ${ }^{[9,10]}$, rely on the centrifugal force of the wind wheel, inertia force when wind turbine emergency stop, vibration of 
wind turbine, pneumatic blowing ice or expansion pipe, etc., the efficiency of de-icing is low and non planned shutdown is needed. C. Ultrasonic de-icing program ${ }^{[11]}$, using Lamb wave and SH wave presented in the ultrasonic to produce the velocity difference on the surface of blade, thus forming the shear stress, to achieve the purpose of removing ice. D. Microwave heating program ${ }^{\text {[12] }}$, the surface temperature of the blade can be promoted by the high frequency microwave, which can prevent or remove the ice cover, but the efficiency, is low and no practical application. E. Thermal de-icing scheme, electric heating ${ }^{[13,14]}$ by resistance heating layer laid to the leaf surface layer or inner surface or the appearance, the thermal resistance electricity heating melting blade surface icing, has a fast reaction speed and high efficiency, but it is required to strictly control the heating time, too long or too short is not conducive to melt ice, and must be added to the lightning protection device, cannot replace the damage resistance, the introduction of technology is expensive. Hot air heating, a blower and a heating device are installed in the blade root by the General Electric Company ${ }^{[15]}$, and the hot air is blown into the inner cavity of the blade to heat transfer with the outside environment., so that the snow melt off, safe operation, simple equipment, low cost, but the consumption of high power electric energy; as for the long blade, hot air cannot reach tip area mainly collecting wind energy from the root, the efficiency is low.

In order to remove the blade surface icing of wind turbine, to make sure the safe, stable and efficient operation, based on the consideration of de-icing effect, safety performance, equipment costs and other comprehensive factors, this paper proposed a new hot air blower technology. By numerical simulation, the flow state of the whole flow field in the blade of the ice removal system is analyzed. The temperature rise of the heat flow field of the blade is calculated, which ensures that the optimization scheme has an obvious de-icing ability for the southern winter weather (about 10 degrees below zero). The constant temperature test chamber test is carried out to compare the consistency between the temperature rise test data and the numerical simulation data, so as to verify the feasibility of the hot air blower de-icing technology.

\section{Design of hot air blower de-icing system for blade}

Determine the blade de-icing area

Blade is the main component of wind turbine to collect wind power, and it also needs to bear the load of wind energy, so the structure of blade should have good aerodynamic characteristics, at the same time, must meet the performance requirements of strength and stiffness. Blade consists of blade root, leading edge, girder, web, trailing edge and tip, the blade root zone bears the maximum load and is designed to be cylindrical and the wall is thick; the leading edge region is the main upwind region from the blade airfoil; the trailing edge region is designed as the thin-walled structure to meet the aerodynamic characteristics; the tip area determines the wind area, will directly affect the size of the wind energy, a typical airfoil structure is shown as figure 1 . Therefore, the de-icing region is determined in the leading edge region where catches the main wind energy, and taking into account the removal of the entire blade icing on the surface of the blade is not realistic and efficient. By increasing the heater vent line, at the same time, between the front edge and the front web plate is mounted on the baffle plate, so the heat energy is kept in the most important area where bears the most wind load. The distribution of wind load of blades is calculated by using the Bladed software, found that the baffle installed in the distance from the root $20 \mathrm{~m}$, the region of baffle to the tip of blade leading edge captured wind energy which will reach about $80 \%$ of the whole blade. Thermal gas flow field of the region from baffle to the tip of blade leading edge conducts heat convection and heat conduction through the blade wall with the surface with cold temperature, to make sure the temperature of ice is up to $0^{\circ} \mathrm{C}$ to melt, this can be more efficient to 
ensure the safe operation and the ability of blade to catch the wind.

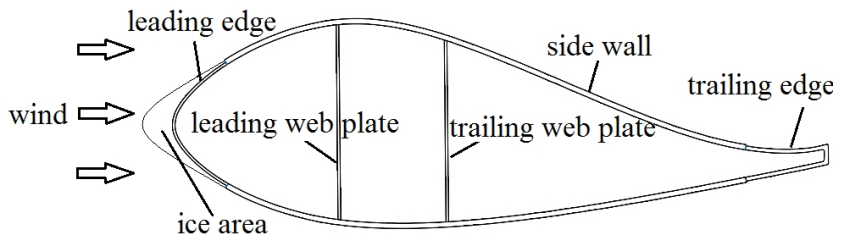

Fig.1. The classic wind turbine airfoil

The whole design of hot air blower de-icing system for blade

The hot air blower de-icing system for blade of wind turbine comprises power distribution cabinet, de-icing control cabinet, blade root control cabinet, blower, heater, temperature sensor, ventilation pipe, baffle, and distribution cables, see figure 2. The basic parameters of the hot air blower de-icing system are shown in table 1 . The working principle is: because the blade is equipped with hot air blower system, through the box converter supply the power of the tower control cabinet, the power cable from the base along the tower connecting with the special electric slip ring to supply power to the de-icing control cabinet, and the de-icing control cabinet supply power to three blade root control cabinets. Root control cabinet controls the opening and closing of blower and heater blower, then the hot air flow through the ventilation pipe is transferred the specified de-icing area, in order to ensure the de-icing efficiency, a baffle is arranged on the positions of blade leading edge and the web, so that the hot air flow is maintained in the important de-icing area, using the exchange of the hot air flow in the blade cavity with the cold air in the blade surface, when the temperature of blade surface rises above zero degrees Celsius, the ice covered in the outer surface of the blade melt into water, so as to achieve the purpose of removing ice. When the pressure of blade cavity rises, the heat flow flows out via the gap of the tip of front and front web, and through the gap between the front and back web or the gap between the trailing web and the trailing edge back to the entrance, so as to realize the air circulation in the inner cavity of blade.

Table 1: Basic parameters of hot air blower de-icing system in blade

\begin{tabular}{cc}
\hline Item & Parameter \\
\hline Blade & TMT56.5m \\
Flow of blower $(Q)$ & $3000 \mathrm{~m}^{3} / \mathrm{h}$ \\
Pressure of blower $(P)$ & $1400 \mathrm{pa}$ \\
Velocity $(v)$ & $12 \sim 14 \mathrm{~m} / \mathrm{s}$ \\
Diameter of pipe $(D)$ & $200 \mathrm{~mm}$ \\
\hline
\end{tabular}

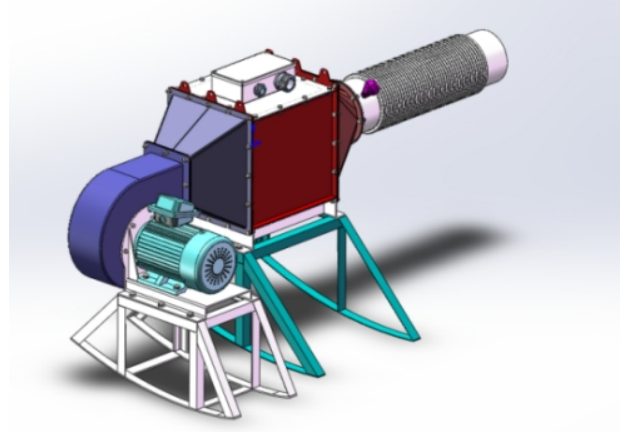

Fig.2. The structure diagram of hot air blower de-icing system

\section{The heat flow field simulation of blade}

The flow field in the blade of de-icing system

Figure 3 is the flow velocity of the whole flow field in the blade of the heat blower de-icing system. From figure 3, we can see that the hot air flow into the circular tube is at the speed of $12 \sim 14 \mathrm{~m} / \mathrm{s}$, and in the whole of the ventilation tube the speed basically remained unchanged. When the air flows out from ventilation pipe into the baffle at the front cavity space, velocity decays rapidly to about $8 \sim 10 \mathrm{~m} / \mathrm{s}$, and the vortex flow generated, the vortex size range from tip to baffle at the direction of 2 meters, see figure 4(a) and figure 4(b), due to the existence of the hot gas flow vortex, the heat gathering will be occurred, so that the temperature of the inner cavity of this area will be 
relatively high. The hot air flow through the vortex of the leading edge, basically changed to the state of the smooth flow, the speed decreased to $4 \mathrm{~m} / \mathrm{s}$. When the air came to the front part of the blade tip, because the gap of the front web and leading edge is sharply compressed, where the speed of airflow rises instantly, through the narrow tip region into the inner cavity of front and the trailing webs, the speed began to decay again, see figure 5. When the air came to the trailing webs and the trailing edge, because the air velocity is relatively small, and the gap between front and trailing web is larger than the gap between trailing web and trailing edge, resulting in air gap at the trailing edge and trailing web formed a small vortex, see figure 6 , to prevent the air flowing into the space of the trailing web, so that the flow along the cavity between the front and the trailing webs returns to the root zone. When the airflow reaches the root of the blade, the air flow is accelerated to about $8 \mathrm{~m} / \mathrm{s}$ and into the blower, and the air flow in the inner closed cavity is an internal circulating, and the expected temperature rise of the leading edge is about $10 \mathrm{~K}$.

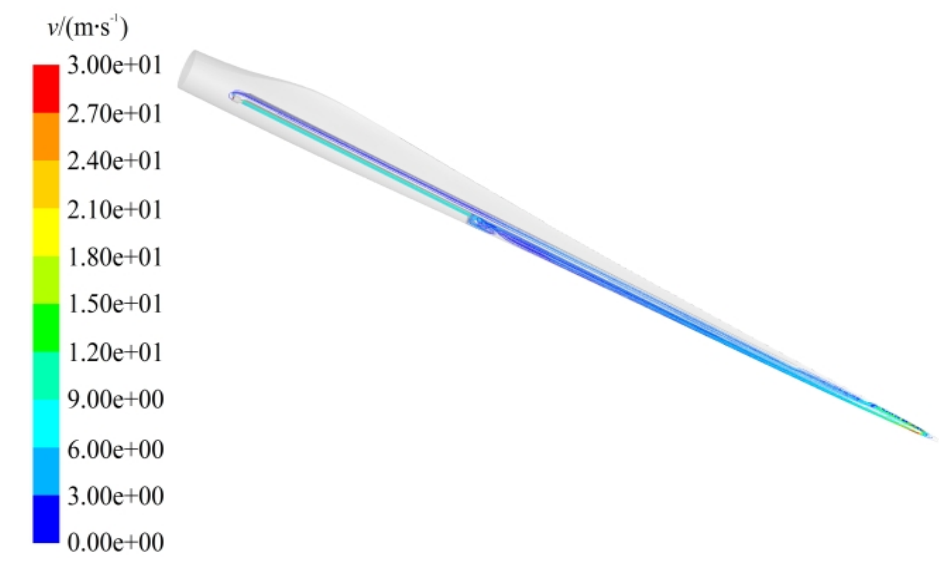

Fig.3. Whole inner flow field in blade

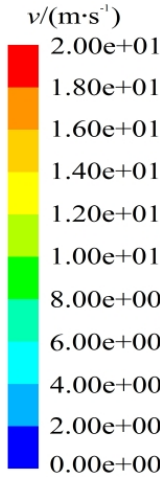

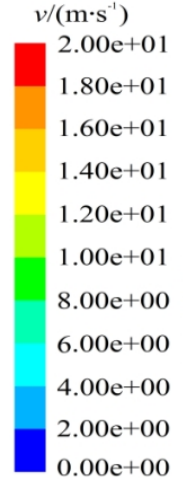

(a) Velocity contour (b) Velocity streamline

Fig.4. Velocity near baffle plate

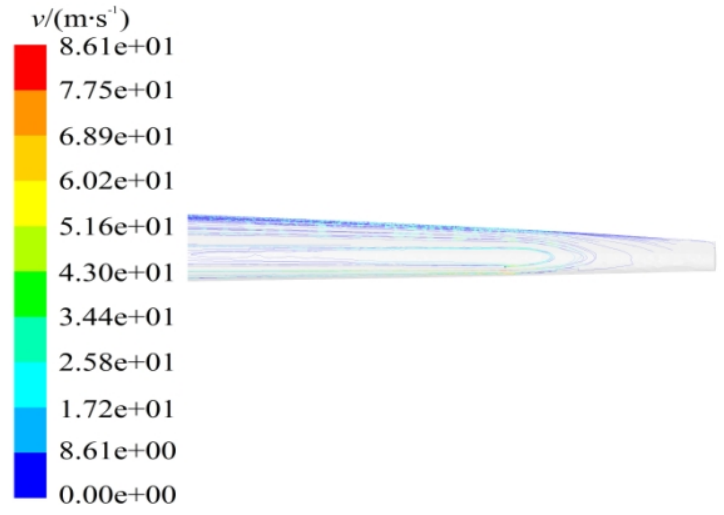

Fig.5. Velocity streamline of blade tip

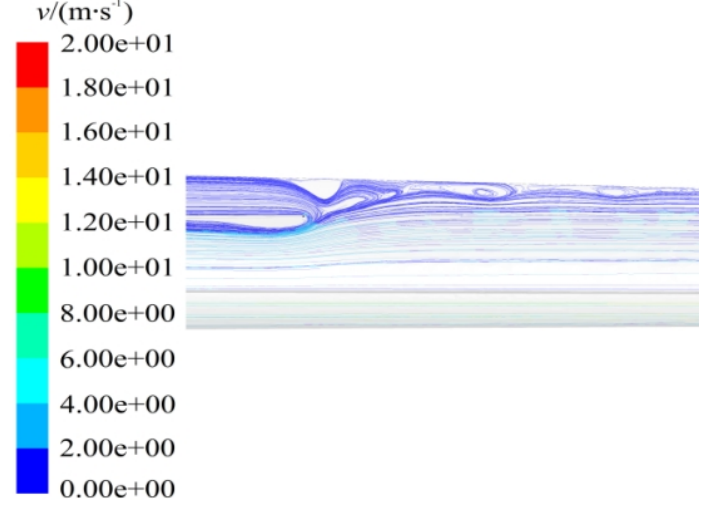

Fig.6. Velocity streamline near trailing edge 
Temperature field in heat transfer process about de-icing system

In order to pursue the reality and reliability of the de-icing system, the $1: 1$ size of the three dimensional modeling of blade is used, the grid number of flow field in the blade is about 6 million. When the heat transfer between the hot air in the inner cavity of the blade cavity with the outside cold air is taken into consideration, considering the calculation accuracy, the size of the external flow field is at least three times the size of the internal flow field, the grid number will reach tens of millions. Considering the amount of data calculation and problem of data processing, in order to solve the contradictions, capture four important sections of the blade: section 1 near the baffle (distance from leaf root 22.6m), and the section 2 between the baffle and the tip region (distance from leaf root $32 \mathrm{~m}$ ) and 3 (distance from leaf root $43.2 \mathrm{~m}$ ), and the section 4 in tail tip abdominal surface (distance from leaf root $54.5 \mathrm{~m}$ ), see figure 7 . Numerical simulation is used to analyze the thermal conductivity of these two-dimensional cross sections, and to understand the temperature rise of the de-icing system. The two-dimensional section of external flow field's arc radius is $1 \mathrm{C}$ (C is the chord length of the longest wing type of all sections of blade about $1927.8 \mathrm{~mm}$ ), both sides of the boundary is $1.5 \mathrm{C}$, internal and external flow fields of two-dimensional section is shown in figure 8 .

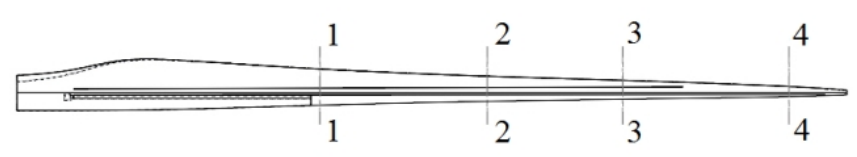

Fig.7. Distribution of section

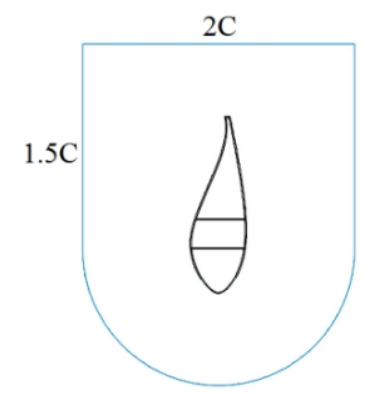

Fig.8. Distribution of internal and external field under $2 \mathrm{~d}$-section

Boundary conditions: Section 1 (wall thickness of front glass steel is10mm, the trailing edge's wall thickness of glass steel is $15 \mathrm{~mm}$, both sides of PVC foam wall's thickness is $20 \mathrm{~mm}$ ), Section 2 (material is the same with Section 1), Section 3 (material is the same with Section 1), Section 4 (material is the same with Section 1, no trailing web). The thickness of PVC foam plate of the leading edge web and the trailing web is $10 \mathrm{~mm}$, outdoor temperature is 30 degrees Celsius, thermal physical parameters of each material are shown in table 2.

Table 2: Material thermo-physical parameter

\begin{tabular}{ccc}
\hline Thermal parameter & GFRP & PVC \\
\hline heat conductivity $\mathrm{W} /(\mathrm{m} \cdot \mathrm{K})$ & 0.45 & 0.16 \\
Specific heat $\mathrm{J} /(\mathrm{kg} \cdot \mathrm{K})$ & 1260 & 900 \\
Density $\mathrm{kg} / \mathrm{m} 3$ & 1800 & 1380 \\
\hline
\end{tabular}

Figure 9 is the temperature cloud picture of heat transfer between the hot air in the Section 1 of blade with the outside cold air. From figure 9(a), the blade inner temperature field is divided into three segments by the front web and the trailing web (leading edge cavity, cavity between the leading edge web and the trailing web, trailing edge cavity), high temperature area concentrated in the leading edge cavity (the area between the leading edge and the front bulkhead), namely is the designated de-icing area. Figure 9(b) is local magnification image of section 1 of the leading edge, the figure shows that due to heat flow from the ventilation pipe, and form vortex in the baffle, resulting in agglomeration of heat flow, the front glass steel outer surface's temperature rises sharply to $322 \mathrm{~K}\left(49{ }^{\circ} \mathrm{C}\right)$, compared to the outdoor ambient temperature $303 \mathrm{~K}\left(30{ }^{\circ} \mathrm{C}\right)$, the temperature rise reaches $19 \mathrm{~K}$ Celsius, the lifting degree is obviously to meet the requirements of 
the basic temperature rise of the de-icing system; Figure 9(c) is local magnification image of the interface between the front web and the side blade, and the interface between the leading edge blade wall and the side blade wall, it can be seen that the heat transfer capability of PVC foam board is obviously not as good as that of glass fiber reinforced plastic. Because the temperature of the front cavity is high, the outer surface temperature of the PVC foam plate is $310 \mathrm{~K}\left(37{ }^{\circ} \mathrm{C}\right)$, and the temperature rise is $7 \mathrm{~K}$, the leading edge cavity transfer heat to a certain temperature to the inner cavity of leading edge web and the trailing web through the leading web, but the trailing edge cavity temperature $328 \mathrm{~K}\left(55^{\circ} \mathrm{C}\right)$ is mainly by hot air exchange by the flows refluxing through the leading edge cavity and then through the tip region, and then through the poor thermal conductivity of the PVC foam board to PVC foam board, the temperature outside the surface is $308 \mathrm{~K}\left(35^{\circ} \mathrm{C}\right)$, the temperature rise is $5 \mathrm{~K}$, thus we can know that the temperature rise of the blade side edge PVC foam board is ordinary. But considering the ice concentration in the leading edge region, and due to the poor thermal conductivity of PVC foam board, the hot gas flow at the leading edge of the leading edge can only be exchanged at the leading edge of the glass fiber reinforced plastic, which will improve the de-icing performance in the leading edge region in a better way. The temperature of the trailing edge cavity (region between trailing web and trailing edge) is $308 \mathrm{~K}\left(35^{\circ} \mathrm{C}\right)$. Because the temperature is low as the flow refluxed back from the tip, then through the heat conduction to the outer surface of the blade, the region is not the key areas to deice so it is not to be considered.

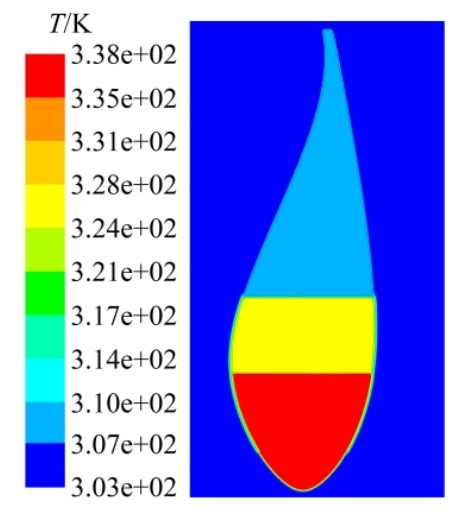

(a) Temperature distribution of each region

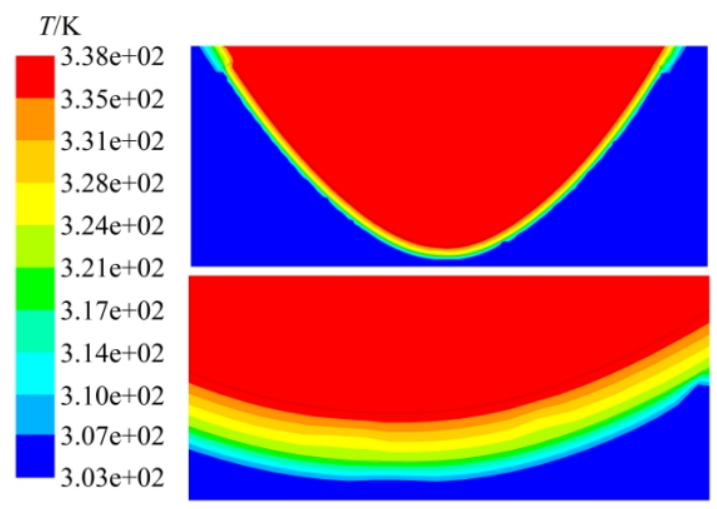

(b) Detail view of leading edge

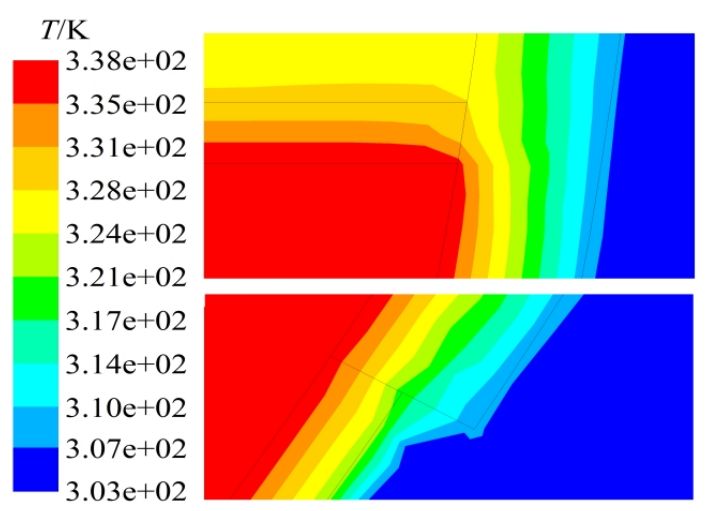

(c) Leading web plate and the interface between front and side

Fig.9. Temperature contour of heat transfer in section 1

Heat transfer temperature contour of blade section 2 is as shown in figure 10, the inner velocity field shows that the heat flow in Section 2 at the leading edge formed the laminar flow, air flows rapidly to heat transfer, so the cavity temperature of section at the leading edge is $331 \mathrm{~K}\left(58^{\circ} \mathrm{C}\right)$, faster drop than the section 1 . The cavity temperature between the leading edge and the trailing edge is $331 \mathrm{~K}\left(45^{\circ} \mathrm{C}\right)$. The figure 10 (b) shows that the outer surface temperature of the section 2 
leading edge of the glass fiber reinforced plastic is $316 \mathrm{~K}\left(43^{\circ} \mathrm{C}\right)$, the temperature rise is $13 \mathrm{~K}$ which can meet the requirements of de-icing. Figure 10(c) shows that due to the cavity temperature of leading edge drops, the temperature of the outer surface of the inner cavity of the front edge of the blade passing through the blade side wall is $307 \mathrm{~K}\left(34^{\circ} \mathrm{C}\right)$, and the temperature of the outer surface of the front and back PVC foam board is $306 \mathrm{~K}\left(33^{\circ} \mathrm{C}\right)$. The side edge can be used as a good thermal insulation wall to prevent heat diffusion, and the heat energy is mainly used for de-icing and melting ice in leading edge to improve the effect of removing ice.

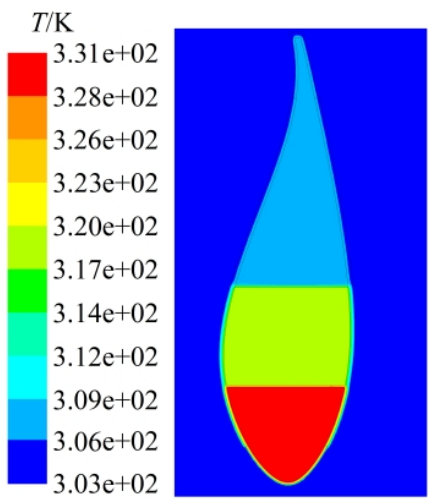

(a) Temperature distribution of each region

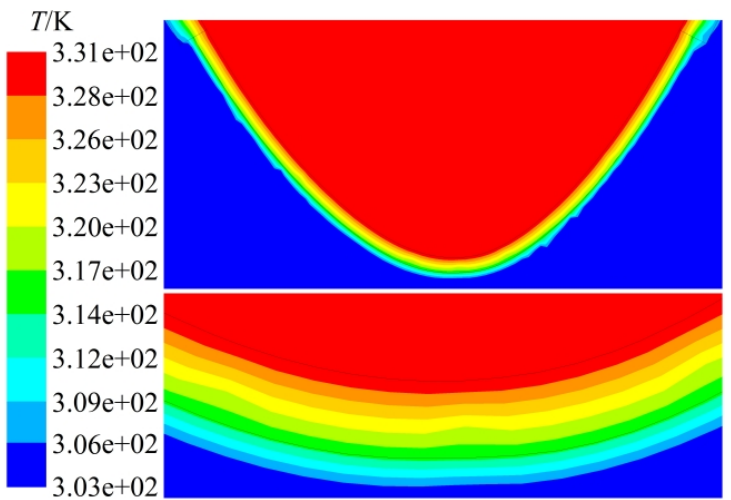

(b) Detail view of leading edge

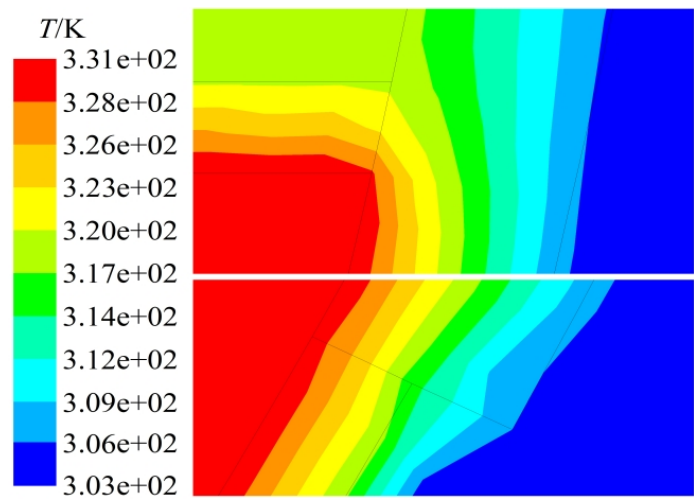

(c) Leading web plate and the interface between front and side

Fig.10. Temperature contour of heat transfer in section 2

Heat transfer temperature contour of blade section 3 is as shown in figure 11, because the structure of the section 2 is similar to the section 3, and the velocity field shows that the flow state is basically the same, which leads to the temperature field distribution of the two is the same and the temperature range is close. From figure 11(a), the cavity temperature of section at the leading edge is $328 \mathrm{~K}\left(55^{\circ} \mathrm{C}\right)$, the cavity temperature between the leading edge web and the trailing edge web is $319 \mathrm{~K}\left(46^{\circ} \mathrm{C}\right)$. Figure $11(\mathrm{~b})$ shows that the surface temperature of the section 3 of leading edge glass steel is $313 \mathrm{~K}\left(40^{\circ} \mathrm{C}\right)$, temperature rise of $10 \mathrm{~K}$ can achieve the basic requirements of the de-icing. Figure 11(c) shows that the outer surface temperature of PVC foam plate side wall in the leading edge and the outer surface temperature of side in the cavity between the leading edge web and the trailing edge web are both $306 \mathrm{~K}\left(33^{\circ} \mathrm{C}\right)$. 


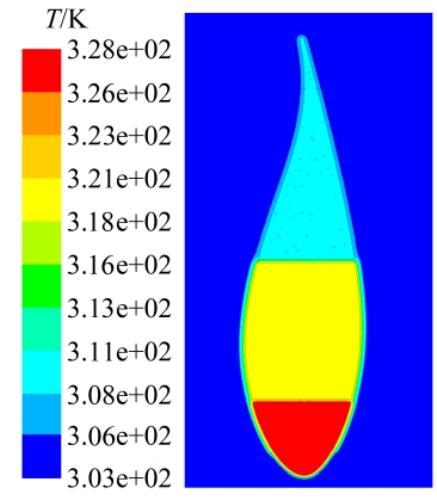

(a) Temperature distribution of each region

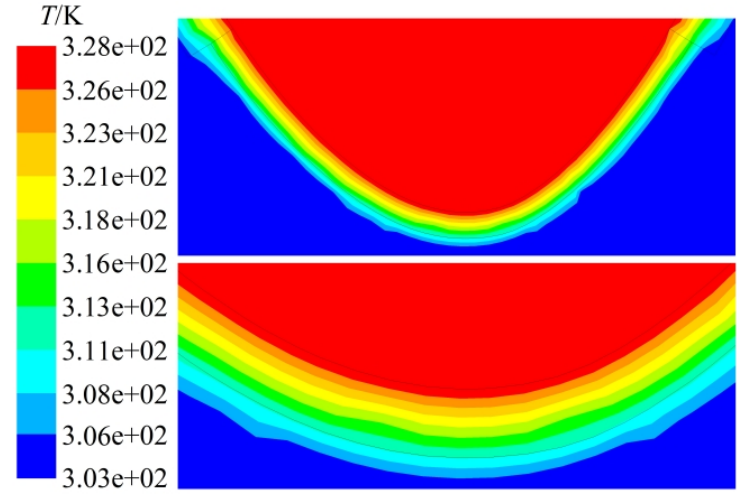

(b) Detail view of leading edge

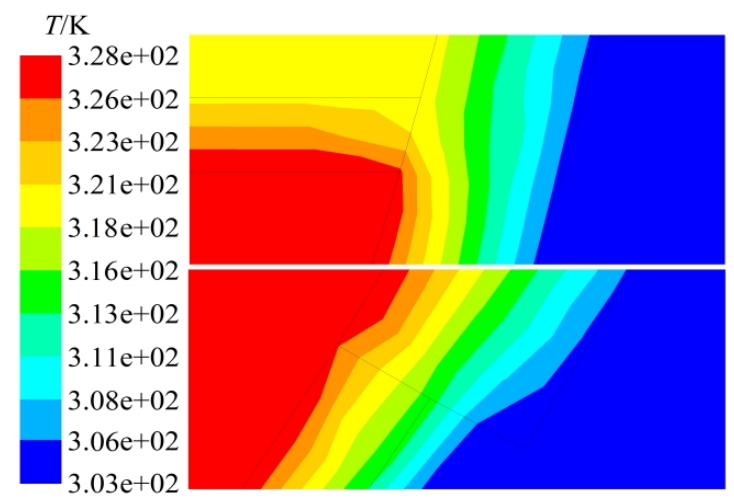

(c) Leading web plate and the interface between front and side

Fig.11. Temperature contour of heat transfer in section 3

Heat transfer temperature contour of the blade section 4 is shown in figure 12, the section is in the tip region, because the former web is longer than the back web, only the front web can be seen in the section. The front web plate, the side edge and the trailing edge form the inner cavity of the trailing edge, and the inner cavity temperature of the leading edge is $323 \mathrm{~K}\left(50^{\circ} \mathrm{C}\right)$, the inner cavity temperature of the trailing edge is $321 \mathrm{~K}\left(48^{\circ} \mathrm{C}\right)$. Because the hot gas flow in the leading edge cavity through the tip region reaches the trailing edge cavity is very fast due to pressure effect, so the temperature of the two is close. Figure 12(b) shows that the outer surface temperature of the leading edge glass steel is $312 \mathrm{~K}\left(39^{\circ} \mathrm{C}\right)$, temperature rise is $9 \mathrm{~K}$, basically meets the function of de-icing system. Fig. 12(c) shows that the outer surface temperature of the side edge PVC foam plate is 306K, the same with the section 2 and section 3, which shows that the thermal insulation performance of PVC foam board is good. For the presence of a certain temperature difference between the inner cavity temperature, when the heat conducts to the outer surface, to ensure a very low thermal diffusivity, so that the heat can be gathered at the leading edge and then diffuses.

The above four sections also indicate that the internal heat energy of the blade is spread out from the leading edge to the outside, although there is a temperature rise difference from the tip to the out surface of baffle area (between $9 \mathrm{~K} \sim 19 \mathrm{~K}$ ), but basically meet the requirements of de-icing, and the good thermal performance of the blade side wall PVC foam board can also further improve the effect of de-icing in the leading edge. 


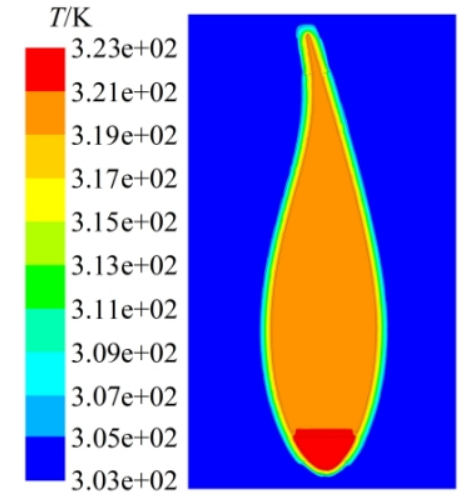

(a) Temperature distribution of each region

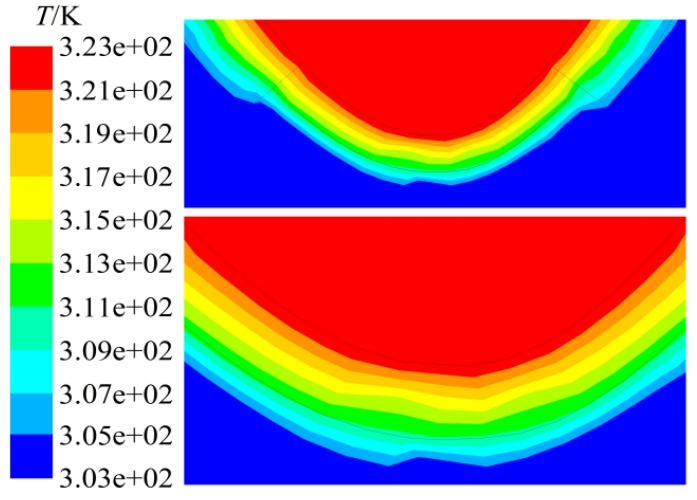

(b) Detail view of leading edge

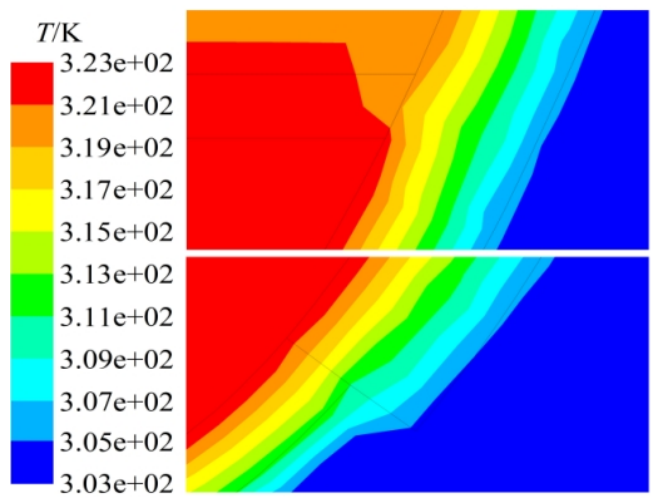

(c) Leading web plate and the interface between front and side

Fig.12. Temperature contour of heat transfer in section 4

\section{Experimental test and data analysis}

Static tests for Blade TMT56.5 were carried out by blade manufacturer in constant temperature laboratory. 1:1 mock up were put to test in order to maintain authenticity and validity of data. The area of blade root was block out so that the flow field inner blade was under self-circulation as the actual situation. Due to the limitation of weather and time schedule, de-icing test was not implemented and the experimental environment was at the constant temperature of $30^{\circ} \mathrm{C}$.

Warm flow ventilator system includes control cabinet, ventilation pipe, heater, temperature sensor, air duct and so forth. As figure 13 shows, control cabinet was out of the blade root, other equipments were installed inside the blade. After the three ventilators were turned on, 4 temperature sensors (PT100), which were placed at outlet of heater, outlet of air duct (at the baffle), cavity near the leading edge ( $22.5 \mathrm{~m}$ away from blade root) and blade tip, all started to record the temperature change inside the blades respectively. Besides, a infrared thermometer and temperature sensors were also used for measuring temperature rise outside the blade, which were showed in figure 14(a) (c). The measurement for outer surface of blades started from a point $21 \mathrm{~m}$ away from blade root and ended at the point $56 \mathrm{~m}$ from blade root. In particular, temperature were measured every $1 \mathrm{~m}$ and thus there are 36 sets (from $21 \mathrm{~m}-56 \mathrm{~m}$ ) of data for each blade. Figure 14(b) shows the temperature distribution using thermal imaging equipment. 


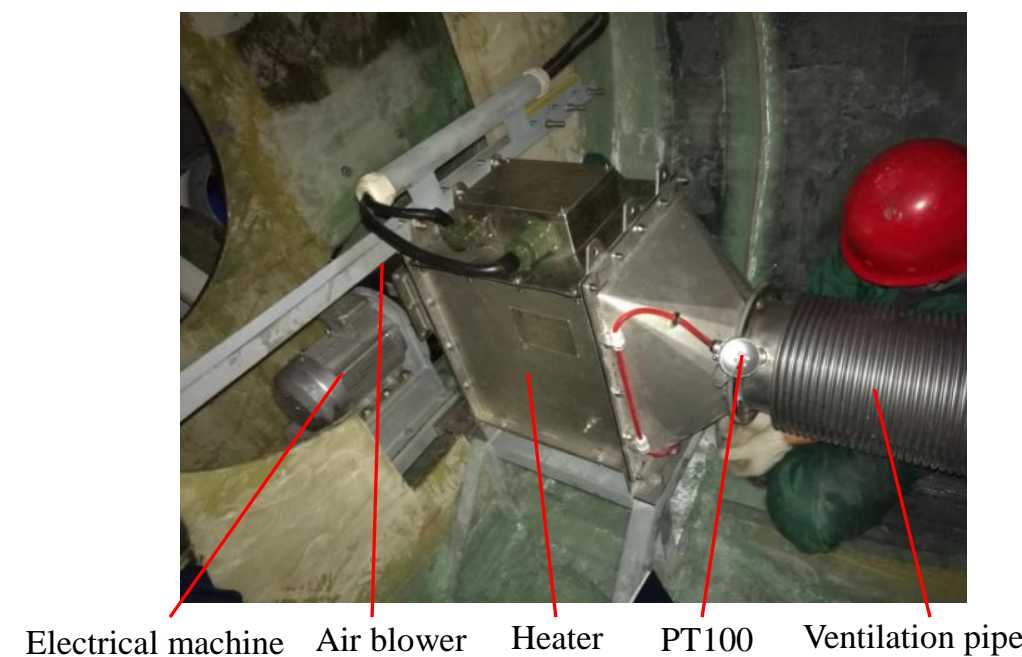

Fig.13. The structure of hot air blower de-icing system

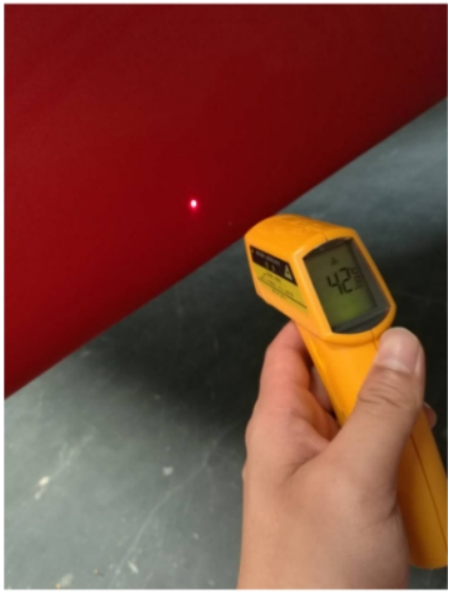

(a) Temperature measuring probe

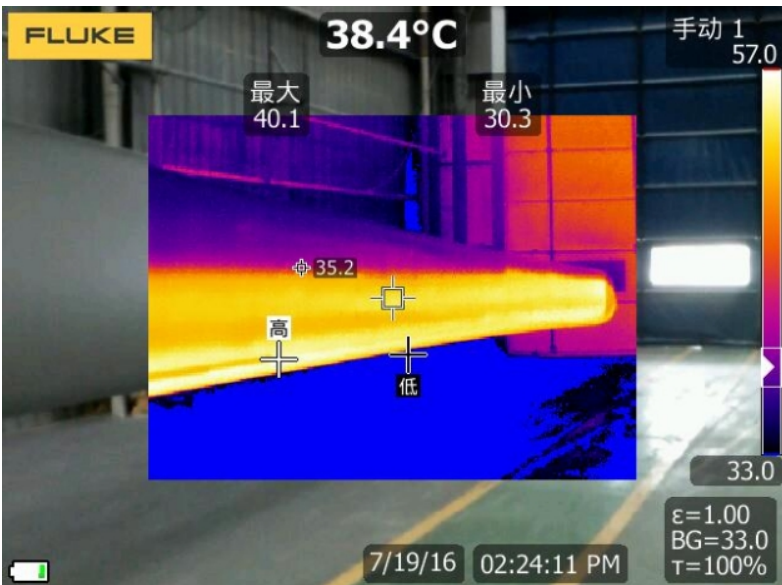

(b) Thermal imager
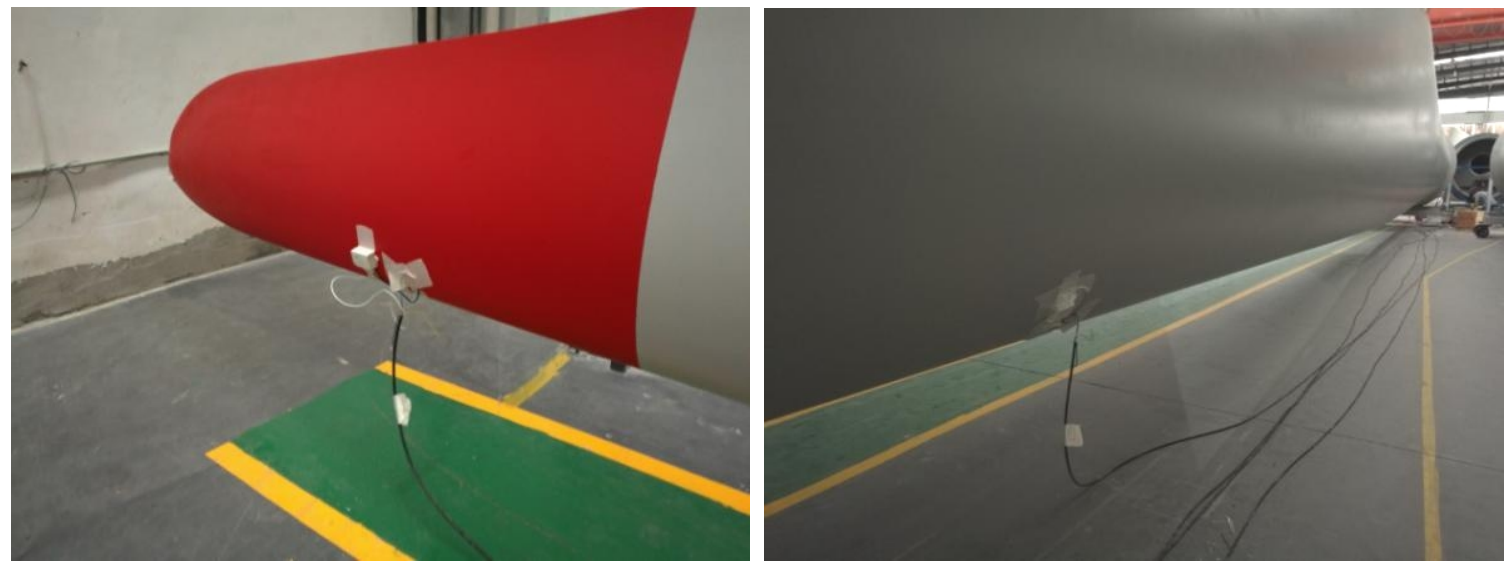

(c) Temperature sensor

Fig.14. Temperature monitoring on the surface of blade

Temperature rise measurement

After de-icing system is turned on, 4 temperature sensors installed in blade cavity collect data every minute and the duration is 1 hour, which means there are 60 sets of data for each sensor. As figure 15 shows, temperature of outlet of heater, outlet of air duct, cavity of blade and blade tip is steady, nearly 70.7, 68.7, 65.3 and 47.2. The simulation result of section 1 (cavity) is 65, which is close to the measured value 65.3; the simulated temperature of leading edge is 50 , which is close to the measurement 47.2 (deviation $5.9 \%$ is acceptable). Due to test limitation, the measurement for section 2 and 3 was not carried out, but the difference between section 2 and 3 is also less than 5.9\%. 
In summary, the simulation results are accord with measurement.

Figure 16 shows the temperature measurement results of leading edge of three blades. It indicates that temperature increases dramatically on the leading edge surface from the baffle to the blade tip. For blade 1 (11.4 16.8), the lowest temperature rise is located at blade tip and the highest rise is $22 \mathrm{~m}$ away from blade root; for blade 2 (11.8 14.8), the lowest temperature rise is at blade tip and the highest rise is placed at $22 \mathrm{~m}$ away from blade root; for blade 3 (11 15.2), the lowest rise is also blade tip and the highest rise is $21 \mathrm{~m}$ away from blade root. The temperature rise situation for each blade is quite similar and the location with lowest and highest temperature rise tallies with the flow regulation in simulation, which proves the accuracy and validity of simulation.

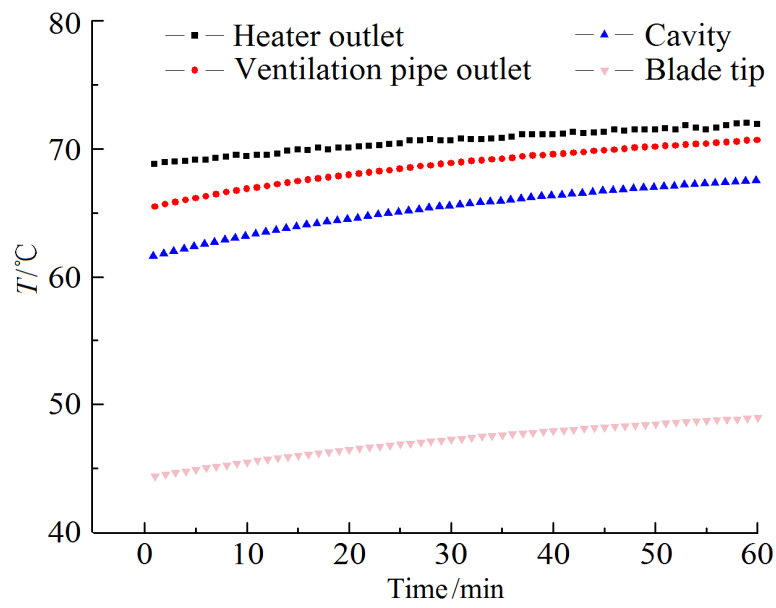

Fig.15. The internal temperature test of blade

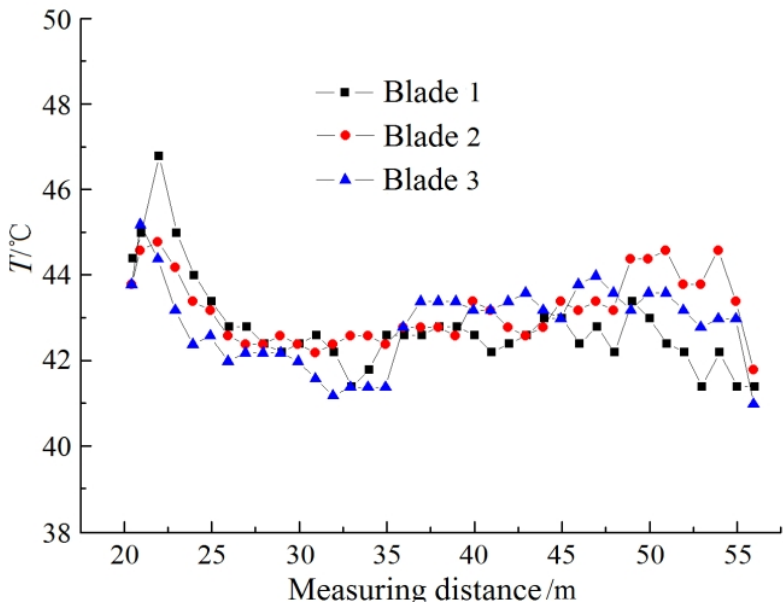

Fig.16. The temperature rising test on surface of leading edge

Figure 17 includes the comparison of simulation and measurement results. As figure 17 shows, simulation results of section 1 and 2 are larger than measurements, while simulation results of section 3 and 4 are smaller than measurements. The reason is as following: as for section 1 and 2, the simulation model was simplified so that the material of leading edge is set as GFRP (Fiber Reinforced Plastics) rather than the composition of GFRP, PVC and air. As the capacity of heat transfer of composite material is worse than pure GFRP, the simulated temperature of section 1 and 2 is higher than measurement; as for section 3 and 4, those places are away from the outlet of flow duct and the actual flow field is not as smooth as the simulation describes, therefore simulation values of temperature are lower than the real ones.

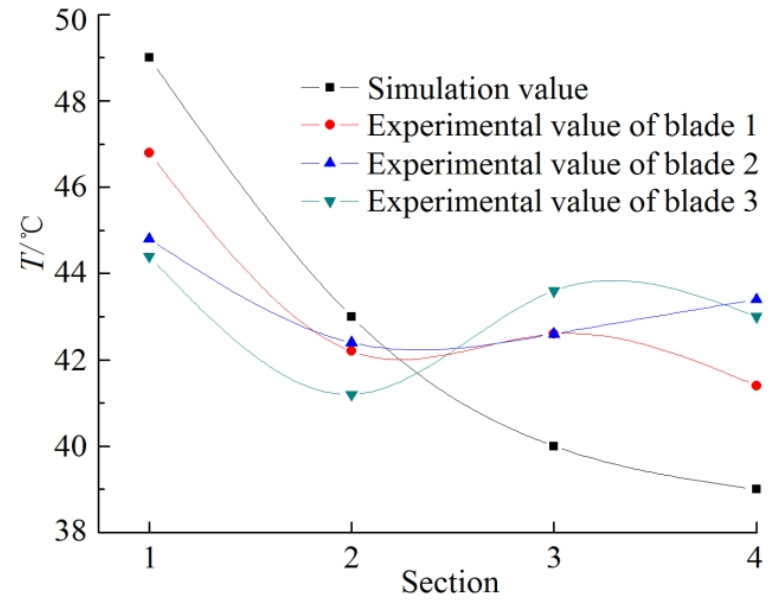

Fig.17. The temperature verification on the surface leading edge

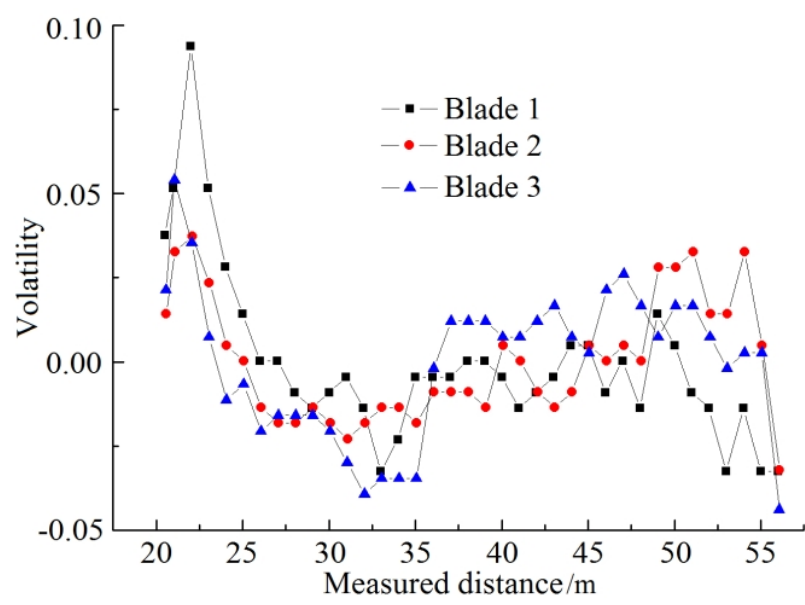

Fig.18. Distribution of temperature fluctuation on the of surface of leading edge

The uniformity of temperature field

Figure 18 shows temperature fluctuation of leading edge's surface of three blades. The tendency of 
temperature fluctuation is almost the same. All the temperature rise at each measurement points fluctuates around average value within limited range: the average temperature of blade 1 is 42.8 with fluctuation range $-3.25 \% \sim 9.37 \%$, that of blade 2 is 43.2 with fluctuation range $-3.24 \% \sim 3.7 \%$ and for blade 3 it is 42.9 within fluctuation range $-4.38 \% \sim 5.41$. The data demonstrates that by using warm air de-icing method, the temperature rise at certain area is uniform and the temperature rise meet the demand of blade de-icing.

\section{Conclusion}

The paper analyzed warm flow distribution around the whole blade profile and heat transfer effects inner and out of blades via numerical simulation. Besides, temperature rise and heat distribution on the blades were measured in constant temperature laboratory. The results are as followed:

1) Warm air flows through pipe and goes into cavity of leading edge. Around the baffle, vortex forms and leads to heat accumulation, which makes the surface of leading edge the best effect of temperature rise.

2) Heat transfer is efficient on the four key sections of blades, and thus temperature increases correspondingly. The simulation values of elevated temperature are from $9 \mathrm{~K}$ to $19 \mathrm{~K}$ and the measurement values are from $11 \mathrm{~K}$ to $16.8 \mathrm{~K}$, of which the difference is only within $8.5 \%$. The high consistency of simulation and measurements confirms that warm air flow method reaches the requirement of blade de-icing.

3) Through comparing the comparison of fluctuating range of temperature rise on three blades, it is found that the maximal and minimal values are both within $9.5 \%$ deviation from the average value, which proves the coherence of three blades. The appropriate heat distribution on leading edge of blades validates the effectiveness of this de-icing technique.

\section{References:}

[1] Li Qiao. Wind power development in our country enter the era of low wind speed [N]. China Industry News, 2012-22-5. (in Chinese)

[2] Hou Binbin. Technology of large-scale anti-icing blades for low wind speed wind turbine [J]. High Power Converter Technology, 2013, (3): 78-81. (in Chinese)

[3] Wang Yijin. Research of anti-icing technique of the wind turbine [J]. Mechanical and Electronic Information, 2011, (9): 91-92. (in Chinese)

[4] Wang Xiangming. The research of low temperature environment on the influence of the wind turbine [J]. Wind Power, 2005, (3): 37-39. (in Chinese)

[5] Clement Hochartm, Guy Fortin, Jean Perron, et al. Wind turbine performance under icing conditions [J]. Wind Energy, 2008, 11(4): 319-333.

[6] Yang Xiuyu. Analysis of wind turbine blades electric heating de-icing process and ice shedding condition [D]. 2015. (in Chinese)

[7] Ilinca A. Analysis and mitigation of icing effects on wind turbines [J]. Wind Turbine, 2009, (8): 177-214.

[8] Wang Jin, Zhao Wenming, Ji Shuangying, et al. Progress of the aircraft anti-icing/de-icing [J]. Aeronautical Manufacturing Technology, 2015, S3: 31-33. (in Chinese)

[9] Lorenzo Battisti. Anti-icing system for wind turbine [P]. United States Patent. 2009, NO. US7637715B2

[10] Anders Bjorck. Icing of wind turbines [R]. Elforsk report, Stockholm, 2003.

[11] Tan Haihui, Li Luping, Jin Panke, et al. Ultrasonic de-icing theory and method for wind turbine 
blades [J]. Proceedings of the CSEE, 2010, 30(35): 112-117. (in Chinese)

[12] Olivier Parent, Adrian Ilinca. Anti-icing and de-icing techniques for wind turbines: critical review [J]. Cold Regions Science and technology, 2011, (65): 88-96.

[13] Chang Shinan, Hou Yaqin, Yuan Xiugan. Influence of periodic electro heating pulse on de-icing surface temperature [J]. Journal of Aerospace Power, 2007, 22(8): 1247-1251. (in Chinese)

[14] John Maissan, JP Pinard. Development and operation in harsh arctic environments [J]. Wind Energy Resarch. 2011.

[15] Lemieux D L. Methods and apparatus for de-icing airfoils or rotor blades [P]. Google Patents. 2007. 